\title{
The Changing Face of Lung Cancer: Survivor Perspectives on Patient Engagement
}

\section{Margaret I. Fitch}

Bloomberg Faculty of Nursing, University of Toronto, Toronto, Canada

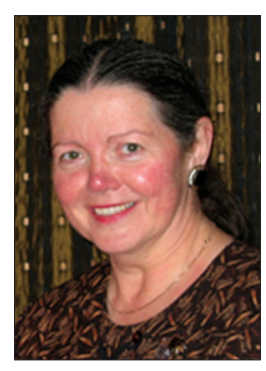

Corresponding author: Margaret I. Fitch, PhD, RN

Bloomberg Faculty of Nursing, University of Toronto, Canada

Tel: +1-416-690-0369

E-mail: marg.i.fitch@gmail.com

Received: July 06, 2018, Accepted: July 20, 2018

\section{A B S T R A C T}

Objective: Advances in the diagnosis and treatment of lung cancer have resulted in an increasing number of individuals living longer following their diagnosis. No longer is lung cancer the "death sentence" it once was. This initiative was designed to document the current experiences of lung cancer patients and explore the potential for patient engagement. Methods: Three avenues of investigation were undertaken: a literature review regarding lung cancer and patient engagement, an environmental scan of lung organizations and cancer societies regarding their approaches to lung cancer patient and family engagement, and in-depth interviews with lung cancer survivors and family members about their experiences and perspectives about patient engagement. Information was collated and major themes identified. Results: Evidence about the experience of lung cancer patients illustrates their needs are complex and dynamic. It also presents a clear picture of unmet physical, psychosocial, and spiritual needs. In particular, stigma is a significant issue for those diagnosed with lung cancer. Information, support, and communication play important roles in helping patients cope but access to resources remains challenging. Patients and family members expressed interest in becoming engaged in advocacy to improve care. Conclusions: The changing face of lung cancer creates the potential for lung cancer survivors to become engaged not only in participating in their own care but also become more involved in peer support and advocacy than lung cancer patients have been able to do in the past.

Key words: Lung cancer, patient engagement, patient experience

\section{Introduction}

The number of individuals diagnosed with lung cancer is expected to double over the next 30 years. Lung cancer accounts for $14 \%$ of all new cancers diagnosed and $25 \%$ of all cancer deaths. ${ }^{[1]}$ However, the context of lung cancer today is complex and evolving. A number of trends in healthcare are currently influencing the experience of

\begin{tabular}{|l|l|}
\hline \multicolumn{2}{|c|}{ Access this article online } \\
\hline Quick Response Code: & \\
\hline & \\
\end{tabular}

individuals diagnosed with lung cancer and their family members. These trends include:

- Increasing availability of screening approaches for lung cancer (for those at high risk)

- Added complexity of treatment protocols including targeted therapies, and resulting alterations in symptoms and side effects profiles

This is an open access journal, and articles are distributed under the terms of the Creative Commons Attribution-NonCommercial-ShareAlike 4.0 License, which allows others to remix, tweak, and build upon the work non-commercially, as long as appropriate credit is given and the new creations are licensed under the identical terms.

For reprints contact: reprints@ @medknow.com

Cite this article as: Fitch MI. The Changing Face of Lung Cancer: Survivor Perspectives on Patient Engagement. Asia Pac J Oncol Nurs 2019;6:17-23. 
- Increasing number of older adults, many of whom have comorbidities,

- Decreasing mortality rates ( $>2 \%$ per year in males) and increasing number of survivors (individuals either cured or living longer with controlled disease), and

- Drive toward person-centered care and patient involvement or engagement in healthcare.

This initiative was undertaken to document the experiences of individuals with lung cancer and to better understand the potential for lung cancer survivors and their family members to engage in activities aimed at improving lung cancer care. Relevant patient/caregiver engagement can result in meaningful intervention/program design and implementation.

\section{Methods}

The project consisted of three parts: A literature review, a series of interviews with survivors and caregivers, and an environmental scan.

\section{Literature review}

The literature review was completed to identify recent literature on supportive care needs of lung cancer patients and their families.

\section{Methods}

Using keywords (e.g., supportive care, patient experience, person-centered care, lung cancer, survivorship) several databases were accessed including Medline, PubMed, and PsychLit to identify literature published in the past 5 years regarding needs of lung cancer patients and caregivers.

\section{Findings}

Supportive care needs apply to all individuals but vary in nature and intensity from one person to the next and over time. ${ }^{[2]} \mathrm{A}$ number of factors influence these needs including life stage, gender, age, and type of disease and treatment. ${ }^{[3-8]}$ In the past, lung cancer patients and their family members have experienced significant levels of unmet supportive care needs. ${ }^{[9-11]}$ Harrison et al. ${ }^{[12]}$ reviewed a series of 94 studies and reported unmet needs in several domains: Physical (7\%-89\%), psychological (12\%-85\%), informational $(6 \%-93 \%)$, spiritual $(14 \%-51 \%)$, sexuality $(33 \%-63 \%)$, and activities of daily living (1\%-73\%). Similarly, Lambert et al. ${ }^{[13]}$ reported unmet needs for caregivers of lung cancer patients following a review of 29 studies: Comprehensive cancer care $(1.1 \%-96 \%)$, emotional/psychological (3\%-93.2\%), impact of activities of daily living $(2.8 \%-79 \%)$, relationships $(3 \%-58 \%)$, informational $(2.2 \%-86 \%)$, and spiritual (2\%-43\%). With the changing dynamics of the lung cancer experience, the nature of these unmet needs may vary.

\section{Environmental scan}

The environmental scan was completed to understanding the current approaches to patient and family engagement by lung and other related health facilities and cancer agencies. Methods

A snowball technique was utilized to identify the relevant organizations involved in lung cancer. Websites for these organizations were reviewed to document how patients and families were engaged with each organization.

\section{Findings}

A trend toward engaging patients and families was clearly identified across healthcare facilities and cancer support organizations. The most frequent approaches involved strategies for engaging individuals in their own direct care decisions. Some organizations have involved patients and families in codesigning programs and delivering educational activities while others had incorporated patients and family members into committees, task forces, and research programs. In the past, relatively few lung cancer survivors have been involved; however, there is a desire to incorporate more as the number of survivors grows.

\section{Interviews with survivors and caregivers}

Interviews with lung cancer survivors and caregivers were conducted to gain insight about the experiences with diagnosis, treatment, and follow-up care.

Methods

Members of the OLA Health Professional Advisory Committee identified individuals in their respective practices whom they thought would be willing to be interview for this project. Eligibility included living in Ontario, a confirmed diagnosis of lung cancer or a caregiver of an individual with lung cancer. Interested individuals were contacted by the project coordinator who explained the project and obtained consent.

Individuals underwent an in-depth interview on the telephone with the project coordinator, individuals with expertise in qualitative research. The interview guide was designed to elicit the experiences of diagnosis and treatment, challenges experienced, and where improvements could be made. The transcripts of the interviews were subjected to a conventional content analysis where content topics were identified and key messages then isolated for each topic. By comparing the key messages across all participants, emergent themes were crafted. These themes were presented to three groups of patients and clinicians to check clarity, relevance, and validity. 
Findings

Twelve individuals completed the semi-structured interview. Eight were lung cancer patients/survivors, and four were family members. The sample reflected males $(n=3)$ and females $(n=9)$, and a range of ages (25-84 years), employment status (employed, retired), and geographic locations across Ontario (i.e., Toronto, Ottawa, Thunder Bay, Southern Ontario).

The findings from these interviews are reported below with reference to the timeline of the cancer journey-from diagnosis to survivorship. Quotes are in Table 1 for illustrative purposes.

\section{Being diagnosed with lung cancer}

The primary challenges participants experienced in relation to being diagnosed with lung cancer included recognizing symptoms as having a potential for lung cancer, timeliness of testing and receiving test results, the emotional shock of hearing the diagnosis, and being upset by the way the diagnosis was communicated. Individuals had symptoms (e.g., pain in back or hip, coughing) but found it took a lengthy period to undergo tests and receive a definitive diagnosis. Hearing the diagnosis was an emotional event with participants experiencing shock, numbness, panic, and disbelief. This was particularly so for individuals who had never smoked. Participants felt the manner in which the diagnosis was communicated, with so much negativity, added to their burden of distress. Participants indicated the "new" messages about lung cancer ought to be clearly communicated early in the course of the treatment discussion: New treatment approaches have resulted in new hope and the emergence of a "new story" about the realities of lung cancer.

\section{Undergoing treatment}

During the treatment interval participants experienced challenges accessing information for decision-making, dealing with side effects, not being able to engage in usual activities, finding support services, and confronting stigma. Participants found that they needed information to help them understand what was happening and make decisions about the next steps they needed to take. For many, they had little information about the disease, its treatment options, or the eventual prognosis at the time of diagnosis.

Once undergoing treatment, the primary challenges related to symptom and side effect management and finding support for their family members. Fatigue was one of the most common and troublesome side effects, interfering with the ability to engage in usual daily activities (i.e., house and yard work, grocery shopping, and driving). Making the decision to continue working through the time of treatment was a consideration. The new types of treatment allow better symptom management than in the past and facilitate oral administration at home. This means it is possible to continue working. Especially for individuals who are self-employed, or those without insurance coverage, this is clearly important financially.

Finding appropriate avenues to obtain emotional support or information about available services in the community was difficult for a number of participants. The lack of professional counselors, especially in rural settings, was a barrier and the availability of in-person support groups for lung cancer patients or survivors was described as "...woefully lacking".

Another significant challenge for survivors was facing the stigma of having lung cancer and others thinking they brought it on themselves by smoking. They found that stigma was evident during their interactions with healthcare providers and members of the general public. Individuals who had never smoked found the judgments made by others particularly troublesome.

\section{Surviving lung cancer}

Both physical and practical changes continue for individuals with lung cancer following primary cancer treatment. Getting "back to normal" and dealing with late effects of treatment were described. Getting back to normal included adjusting to how the body feels and functions, shifts in relationships with others, and re-engaging in the normal activities of life. For some individuals, the cancer diagnosis stimulated a change in attitudes about life and a refocusing on what was important to them.

The profile of late effects for lung cancer survivors is, in some ways, just beginning to be documented in relation to their impact on quality of life. Fatigue may continue after treatment for varying periods of time. Shifts in the ability to learn and recall information as well as concentration were highlighted. Numbness or tingling in hands and feet for many interfered with balance, walking, and their ability to do usual activities. Moreover, an ever-present psychosocial issue was living with the fear of recurrence. Of note, the individuals taking targeted therapies expressed a delight at how well they were feeling and how much they could do each day.

\section{Living with advanced disease}

Those facing advanced disease situations found symptom management and family support were of primary concern. Advancing disease resulted in deteriorating capacity for individuals and an increasing need for physical and practical support in the home. Family members were often the primary caretakers. However, the workload required and the need to balance aspects of the caregiver's own life made for a challenging time. 


\section{Table 1: Examples of quotations to illustrate themes}

\section{Phase of cancer Themes regarding challenges Illustrative quotes \\ trajectory from lung cancer survivors and caregivers}

Being diagnosed Recognition of symptoms as lung We lost two years treating what everyone thought was sciatica...the tumor was not seen until an MRI was cancer done (wife, caregiver)

"The symptom was really not typical for lung cancer, pain in my back, and it took ages to go through the tests and see what was really going on"

His cough and shortness of breath was dismissed at first. He was not a smoker and we didn't even think about lung cancer (wife, caregiver)

We were shocked at the diagnosis. We had never even considered the possibility of lung cancer. Lung cancer is a smoker's disease and he had never smoked (wife, caregiver)

Timeliness of tests and receiving I had a pain in my diaphragm. First, I was told it was a pulled muscle; then an infection, and when it didn't go results away, that the wrong antibiotic had been used...I waited 6 months to see the specialist and by then he said he couldn't do anything. It was too late (female patient)

I had a cough and it was treated with two or three antibiotics before anything else was investigated. It was 8 months, I think, before I was really diagnosed. And by that time, it was inoperable (female patient) It took a year to finally make the diagnosis. Looking back, I feel like I really lost out on a year I could have been getting treatment...the first spot on the X-ray was interpreted as having to do with my COPD, maybe scar tissue (female patient)

Emotional "shock" of the diagnosis

Way the diagnosis was communicated

Need for information about treatment treatment options

Dealing with the demands of treatment (driving every day, symptoms and side effects)

Not being able to work/do usual activities
It is really a life-altering moment. Everything changes (male patient)

We were completely blindsided by the whole thing; complete and utter shock is an understatement (wife) There is an emotional aspect to cancer, to be diagnosed - especially when there is a long drawn out process for getting diagnosed (female patient)

I never smoked a day in my life. How could this be happening to me...was it because Dad smoked all those years ago? (female patient)

Negative messages

I really think she ought to have been more sympathetic when she told me...I went back a month later and told her I did not appreciate how she told me...we were all so very worried and it was not necessary in the end...She told me to make my will and plan my funeral (female patient)

How healthcare professional communicate and the words they use, that is an issue. I would say stay away from using the word "'palliation” at the first diagnosis. I had a shock already (with the diagnosis) and then heard the word palliative and had another. He presented lung cancer as a death sentence (female patient) The way health professionals talk it is like lung cancer is a death sentence from the beginning...to be told by a doctor, why bother with support groups for lung cancer patients because most will be dead in 4 or 5 years, is not helpful (female patient)

Positive messages

People need to know it is not an immediate death sentence like it used to be...it's a different story now (female patient)

Lung cancer is a good news story now. There are plenty of hopeful cases...there are possibilities for a longer life.... a productive life...it is not a death sentence (male patient)

There can be more winners now with lung cancer...you can be a winner and survive (male patient)

When I was diagnosed, I really did not know much about lung cancer. I was just told the diagnosis and that was it (male patient)

...there was not much information around about lung cancer...you are really on your own to find it (female patient)

It was really a very steep learning curve for me (after the diagnosis). It was all so new for me...all the words they use are hard to understand (male patient)

I understood the lung cancer was inoperable, but what else was available and how much benefit would that have, that was what I needed to know (female patient)

I really wanted to see about what clinical trials are open to me... and I wondered about testing for genetics and biomarkers (male patient)

I drove back and forth each day - it was $400 \mathrm{~km}$ roundtrip...At first it was OK, but my biggest concern was the fatigue. I worried about falling asleep while I was driving (male patient)

Sometimes, you feel so overwhelmed, you can forget to breathe (female patient)

I really could not do my usual things around the house or the yard, I just did not have the energy for anything (male patient)

I lived too far away to go back and forth every day, so I stayed in the city. It gets costly and you are away from your family...I worried about what was happening at home (female patient)

Especially if you live alone, you get so very, very tired with treatment, you need someone to check in on you and to help with the practical things, like getting the groceries. I could go to the store if someone drove me, but then I couldn't lift the bags of groceries (female patient)

There needs to be some sort of infrastructure supporting the family...they need to know how to help...I felt like a burden to them (male patient)

Probably the toughest thing for me was not working... and wondering if and when I go back (male patient) I had to stop working for a while and took a hit financially as I am self-employed...found it hard to get back on my feet again when I went back...even now I am not at my old level (male patient) 


\section{Phase of cancer Themes regarding challenges Illustrative quotes}

trajectory from lung cancer survivors and caregivers

Finding support services/groups It's so frustrating. There were times when I wanted to talk with someone about lung cancer and there was nowhere to turn...no one to talk to (female patient)

A major gap is that there are no groups for lung cancer patients...you feel so alone and have nowhere to turn...I attended a support group when I had breast cancer and I know how helpful it can be (female patient) There are few supports for lung cancer patients and none for families. They are coping too and trying to help a loved one get through a tough time. They need support too (male patient)

I was not always sure how to get the help we needed or who to call? (daughter)

Facing the stigma of having lung The first thing everybody seems to ask is did I smoke, you know, when they hear it is lung cancer. And I never cancer

\section{smoked a day in my life! (female)}

You are given a horrific diagnosis and now have to deal with the stigma...now you are being judged by everyone...not asked how you are doing, but did you smoke?.it really adds to the burden you feel...it is the biggest issue for me (female patient, nonsmoker)

It's like you deserve to have lung cancer...it's like they think, what did you expect if you smoked?.so you are embarrassed to tell others (female patient)

Managing side Fatigue, shortness of breath, effects pain, cough, difficulty sleeping, difficulty concentrating, menopause, skin changes, anxiety, bowel changes, sexual changes, nausea/vomiting, hair loss, weight change, neuropathy...

It's not just one thing, you know, it's a number of things all happening together. I am tired but the pain means I can't sleep soundly. I wake up tired and I don't want to do anything, but I am bored. I'm used to being busy at work, but right now I cannot focus on that and do not have the energy for it (male) I had shortness of breath; gained approximately $45 \mathrm{lbs}$ during treatment; ended up with neuropathy in my feet and legs; difficulty walking especially up and down stairs. I also ended up with tendinitis in both ankles and was told not to walk for 6 months or I would end up with casts since it was so severe. Trying to tackle housework and prepare nutritional meals was impossible because I was so tired. I felt help could of/should of been available (female)

I eventually got the help I needed for the pain, but a lot I figured out on my own (female)

Once treatment Dealing with the long-terms is finished effects of treatment and stigma

"It's a good thing I do not have to work right now, I could never hold a (work instrument) with these hands or stand all day with these feet. I am really unsteady when I walk and when I stand, it takes me a little time before I can get going (female patient)

There really is a difference in the way people react to you if you have breast or prostate cancer and if you have lung cancer...You get so you hesitate to volunteer the information that you have lung cancer or reach out for help because of the reaction you get (female patient)

The team at the cancer center was wonderful. They were so supportive when I went there, asking me how I was doing.... once I finished there I felt a little disconnected (female)

You are walking around and looking healthy; people hardly can believe that you have lung cancer (male)

Getting back to "normal" Getting back to normal after - you thought you had a death sentence and now the treatment is a success, and now you have to come back, to adjust, both you and the family. It's not easy

Lung cancer is a good news story now. There are plenty of hopeful cases now...there are possibilities for a longer life.... a productive life. It is not a death sentence (male)

I can see how finances would be a worry for some patients, especially after while if they are not able to work or do not have a coverage plan of some sort there could be a drain on the family finances (male patient) There are side effects that come a few years after treatment that affect your quality of life. And they depend on which treatment you had, targeted therapies or chemo: edema, forgetfulness, memory loss, weight gain, arthritis, skin rashes, digestive changes...you have to learn to live with the disease after treatment...figure what it all means, what to expect, what questions to ask, what you need now (female patient) Getting back to normal afterward - you thought you had a death sentence and now the treatment was a success and now you have to come back, to adjust... both you and your family...it's not so easy...you're entire world turns upside down...life is on hold for a while....and it's hard to get back to it all (male survivor)

Living with uncertainty and fear The mental challenge of accepting that the cancer was gone and was not coming back, the constant fear of it of it coming back

\section{returning}

After months and months of surgery, chemotherapy, radiation, visits to cancer center that it ended abruptly and I was just sent back to my life again. I was fearful and felt there was no one to talk to about it No direction as to potential way to reduce the chance of the cancer returning and what to do, how to navigate (male)

Living with Dealing with symptoms advanced Dad really wanted to stay at home and not go to hospital and we wanted to make sure that happened. But it was hard on all of us, but especially on Mom. She was pretty isolated from her friends and normal activities (daughter)

She is at a point she really cannot stay by herself any more. Her reactions are really slow and she is not thinking clearly. I am afraid she will fall and not be able to get up or something else will happen...we have to organize the family because it is a weekend and we can't get other help right now (sister)

Obtaining information about and Dad is deteriorating now and I think we need more support, but I am not sure how to get that help. Who do I access to services (especially for call? (daughter)

care and support at home)

Caring for someone at home (who is dying) is 24/7. It's like having two full time jobs. I couldn't have managed work and looking after him on my own if my daughter had not moved back home (wife, caregiver)

Navigating the system We weren't sure about where to go for help, who we could ask about things (daughter) It would be very helpful if we had a toolkit to help us navigate our way through (female) 
Table 1: Contd.

Phase of cancer Themes regarding challenges Illustrative quotes

trajectory from lung cancer survivors and caregivers

Balancing life situation/demands I went to a program and learned a lot about breathing exercises and pacing myself (female patient) Balancing my life, with my children and my household and my work, as well as supporting mom and dad and meeting their needs for care, it was a real balancing act (daughter)

\begin{tabular}{ll}
\hline & \\
\hline Cancer & Living through transitions \\
survivorship & Living with uncertainty \\
is filled with & Finding a "new" normal \\
challenges & Living with loss
\end{tabular}
Overarching perspective from lung cancer survivors

My biggest challenge was trying to determine who is coordinating my care following surgery! Who do I call when I am feeling hopeless, depressed, and just having "bad day". I was not provided any support resources. Lack of emotional support for returning to work. Making self-referrals for postoperative complications (physio, footwear, etc.) (female)

Facing uncertainty

Some days you can sort of forget about it and go on with whatever you're doing. Other days not so much. You hear a news story or someone tells you about someone who was just diagnosed and it all comes rushing back...you feel a little like you cannot control it. You just have to learn to live with it, with the uncertainty (female patient)

New ways

You have to adjust to a new way of breathing. I never felt my lungs before...I have to think about breathing now...I had to learn new ways of breathing and doing activities, even like speaking (male patient)

I find I have to pace myself when I garden. I cannot lift things the same or stay out as look without a break.

I get tired and short of breath (female patient)

Targeted therapies

Targeted therapies can be "healthy"', let you live life and do things, have a full life. You just have to watch your energy levels and what you want to do each day...I really have no symptoms and feel like a million dollars now

Dad started on [name of medication] and he was like a new man - he had a new lease on life. He and Mom went traveling, it was unbelievable to see the difference. He had a wonderful 5 years (daughter)

New normal

Trying to go back to the person I was but really didn't exist anymore and realizing that would never happen. It changes who you are and how you look at the world and life (female)

I was lost and scared. You spend all your energies on treatment. When it was over I felt overwhelmed not knowing what to do (female)

I was trying to plan my life and, to make a long story short, I was trying to figure out if I should invest my time in rest and healing with focus on the long-term or stay very active and make memories for my family in the short-term. I'm a realist and I want to balance my life according to both possible outcomes (male) Things have changed regarding my memory and attention span so I've had to adapt my life to these changes. I'm also a lot more emotional. When I think back to what l've been through gets me teary-eyed (female)

Access to palliative care expertise and support was cited as a challenge for individuals remaining at home during the last days of life. When a palliative care service or home care was engaged, it was most helpful to patients and family caregivers. However, when this service was not available, or family members did not have information about how to access the service, the family members felt that the symptom management for patients and support for themselves was sorely lacking. This was a particular issue for those living in small towns or rural settings.

\section{Conclusion}

The lung cancer experience is complex and evolving. Lung cancer is not the "death sentence" it once was as significant treatment changes are emerging. It is important to understand how these changes are experienced by individuals diagnosed with the disease if appropriate support is to be made available to them. Clearly timely diagnosis, access to information, access to support, and access to palliative care remain areas of unmet need. However, as more individuals join the ranks of survivors, more attention needs to be paid to their unique needs related to stigma, fear of recurrence, and management of late effects of treatment. Finally, of particular note, is the growing cadre of individuals who have been diagnosed with lung cancer, and are now survivors, who are in a position to provide feedback about their experiences and thus contribute to improving patient care and support.

\section{Acknowledgments}

This article was written on the basis of a presentation given at MASCC 2018 Conference, Vienna.

\section{Financial support and sponsorship}

This study was supported by Ontario Lung Association.

\section{Conflicts of interest}

There are no conflicts of interest.

\section{References}

1. Canadian Cancer Society. Canadian Cancer Statistics - 2018. Toronto: Canadian Cancer Society; 2018.

2. Fitch M, Porter H, Page B. Supportive Care Framework: A Foundation for Person Centred Care. Pembrook, ON: Papin 
Communications; 2009.

3. Bayly JL, Lloyd-Williams M. Identifying functional impairment and rehabilitation needs in patients newly diagnosed with inoperable lung cancer: A structured review. Support Care Cancer 2016;24:2359-79.

4. Hoeck B, Ledderer L, Hansen HP. Involvement of patients with lung and gynecological cancer and their relatives in psychosocial cancer rehabilitation: A narrative review. Patient 2015;8:127-43.

5. McDowell ME, Occhipinti S, Ferguson M, Dunn J, Chambers SK. Predictors of change in unmet supportive care needs in cancer. Psychooncology 2010;19:508-16.

6. Missel M, Pedersen JH, Hendriksen C, Tewes M, Adamsen L. Diagnosis as the first critical point in the treatment trajectory: An exploration of operable lung cancer patients' lived experiences. Cancer Nurs 2015;38:E12-21.

7. Pozo CL, Morgan MA, Gray JE. Survivorship issues for patients with lung cancer. Cancer Control 2014;21:40-50.

8. Rohan EA, Boehm J, Allen KG, Poehlman J. In their own words: A qualitative study of the psychosocial concerns of posttreatment and long term lung cancer survivors. J Psychosoc Oncol 2016;14:1-15.

9. Fitch MI, Steele R. Supportive care needs of women with lung cancer. Can Oncol Nurs J 2008;18:5.

10. Fitch MI, Steele R. Supportive care needs of individuals with lung cancer. Can Oncol Nurs J 2010;20:15-22.

11. Steinvall K, Johansson H, Berterö C. Balancing a changed life situation: The lived experience from next of kin to persons with inoperable lung cancer. Am J Hosp Palliat Care 2011;28:82-9.

12. Harrison JD, Young JM, Price MA, Butow PN, Solomon MJ. What are the unmet supportive care needs of people with cancer? A systematic review. Support Care Cancer 2009;17:1117-28.

13. Lambert SD, Harrison JD, Smith E, Bonevski B, Carey M, Lawsin $\mathrm{C}$, et al. The unmet needs of partners and caregivers of adults diagnosed with cancer: A systematic review. BMJ Support Palliat Care 2012;2:224-30. 